\title{
Charm Production in Diffractive DIS and PHP at ZEUS
}

\author{
Isabell-Alissandra Melzer-Pellmann, on behalf of the ZEUS collaboration \\ DESY, ZEUS group \\ Notkestr. 85, 22607 Hamburg, Germany
}

\begin{abstract}
The ZEUS experiment has measured charm production in diffractive DIS and in photoproduction. The data are in agreement with perturbative QCD calculations based on various parameterisations of diffractive parton distribution functions. The results are consistent with QCD factorisation in diffractive DIS and direct photoproduction [1].
\end{abstract}

\section{Introduction}

In diffractive electron-proton scattering, the proton loses a small fraction of its energy and either emerges from the scattering intact, $e p \rightarrow e X p$, or dissociates into a low-mass state $N, e p \rightarrow e X N$. Hadronic states $X$ including a $c \bar{c}$ pair are a particularly interesting component of diffractive interactions. The charm-quark mass provides a hard scale, ensuring the applicability of perturbative QCD even for small photon virtualities (photoproduction). At leading order (LO) of QCD, two types of photoproduction processes can be distinguished: direct and resolved photon processes. Charm production mainly proceeds via direct photon reactions, in which the exchanged photon participates as a point-like particle, directly interacting with a gluon from the incoming proton (photon-gluon fusion). Thus, diffractive charm production is directly sensitive to the gluon content of the diffractive exchange. In resolved photon processes, the photon behaves as a hadron-like source of partons, one of which interacts with a parton from the initial proton.

\section{Diffractive $\mathrm{D}^{*}$ in photoproduction}

ZEUS has recently measured diffractive $D^{*}$ in photoproduction [2] in the kinematic range $Q^{2}<1 \mathrm{GeV}^{2}, 130<W<300 \mathrm{GeV}$, with transverse momentum $p_{T}\left(D^{*}\right)>1.9 \mathrm{GeV}$ and pseudorapidity $^{\mathrm{a}}\left|\eta\left(D^{*}\right)\right|<1.6$, using an integrated luminosity of $\mathcal{L}=78.6 \mathrm{pb}^{-1}$. Diffractive events were identified by a large gap in pseudorapidity between the produced hadronic state and the outgoing proton, $\eta_{\max }<3$, reducing the fraction of proton momentum carried by the Pomeron to $x_{\mathbb{P}}<0.035$. In addition, the energy deposited in the forward plug calorimeter (FPC), installed in the $20 \times 20 \mathrm{~cm}^{2}$ beam hole of the forward uranium calorimeter, was required to be consistent with zero $\left(E_{\mathrm{FPC}}<1.5 \mathrm{GeV}\right)$. After all selections, a signal of $458 \pm 30 D^{*}$ mesons was found. In order to reduce the contributions from non-diffractive background, the selection was also performed in the restriced range $x_{\mathbb{P}}<0$.01, where $204 \pm 20$ $D^{*}$ mesons were observed. Proton-dissociative events can also satisfy the diffractive selection requirements if the proton-dissociative system, $N$, has an invariant mass small enough to pass undetected through the forward beam-pipe. The fraction $\left(f_{\mathrm{pd}}\right)$ of these events was measured previously to be $f_{\mathrm{pd}}=16 \pm 4$ (syst.) $\%$ [3].

Cross section predictions for diffractive photoproduction of $D^{*}$ mesons were calculated at the next-to-leading order (NLO) in $\alpha_{s}$, using the fixed-flavour-number scheme, in which only

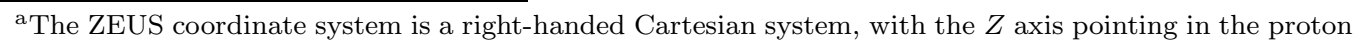
beam direction, referred to as the "forward direction", and the $X$ axis pointing left towards the centre of HERA. The coordinate origin is at the nominal interaction point.
} 
light flavours are active in the parton distribution functions (PDFs) and the heavy quarks are generated by the hard interaction. The calculation was performed with the FMNR code in the double-differential mode $[4,5]$. The Weizsäcker-Williams approximation [6] was used to obtain the virtual photon spectrum for electroproduction with small photon virtualities. Diffractive parton distribution functions (dPDFs) were used instead of the conventional proton PDFs. The three sets of dPDFs used in the calculations were derived from NLO QCD DGLAP fits to the HERA data on diffractive deep inelastic scattering: the H1 2006 Fit A, Fit B [7] and the ZEUS LPS+charm Fit [8] dPDFs. In the ZEUS LPS+charm Fit, the diffractive DIS data were combined with the results on diffractive charm production in DIS [3] to better constrain the gluon contribution. The Reggeon contribution, which amounts to less than $2 \%$ for $x_{\mathbb{P}}=0.01$ and grows up to $\sim 15 \%$ at $x_{\mathbb{P}}=0.035$, was not included. To account for the proton-dissociative contribution, present in the H1 2006 fits, the corresponding predictions were multiplied by the factor 0.81 [7]. The calculations were performed with $\alpha_{s}\left(M_{Z}\right)=0.118 \mathrm{GeV}[9]$ and $m_{c}=1.45 \mathrm{GeV}$, the same values as used in the QCD fits to the HERA data. The fraction of charm quarks hadronising as $D^{*}$ mesons was set to $f\left(c \rightarrow D^{*}\right)=0.238$ [10]. The Peterson parameterisation [11] was used for the charm fragmentation with the Peterson parameter $\epsilon=0.035$. The uncertainties of the calculations were estimated by varying the renormalisation and factorisation scales simultaneously with the charm mass to $\mu_{R}=\mu_{F}=0.5 \cdot \mu, m_{c}=1.25 \mathrm{GeV}$ and to $\mu_{R}=\mu_{F}=2 \cdot \mu, m_{c}=1.65$ $\mathrm{GeV}$. Uncertainties on the dPDFs were not included.

The differential cross section for $e p \rightarrow e D^{*} X^{\prime} p$ in a given variable $\xi$ was calculated from

$$
\frac{d \sigma}{d \xi}=\frac{N_{D^{*}} \cdot\left(1-f_{\text {non-diffractive }}\right) \cdot\left(1-f_{\text {proton-dissociation }}\right)}{\mathcal{A} \cdot \mathcal{L} \cdot \mathcal{B} \cdot \Delta \xi},
$$

where $N_{D^{*}}$ is the number of $D^{*}$ mesons observed in a bin of size $\Delta \xi$. The overall acceptance was $\mathcal{A}=13.9 \%$. The combined $D^{*} \rightarrow\left(D^{0} \rightarrow K \pi\right) \pi_{s}$ decay branching ratio is $\mathcal{B}=0.0257 \pm$ 0.0005 [9]. The measured cross sections are

$$
\begin{aligned}
\sigma_{e p \rightarrow e D^{*} X^{\prime} p}\left(x_{\mathbb{P}}<0.035\right) & \left.=1.49 \pm 0.11 \text { (stat. })_{-0.19}^{+0.11} \text { (syst. }\right) \pm 0.07 \text { (p.d.) nb, and } \\
\sigma_{e p \rightarrow e D^{*} X^{\prime} p}\left(x_{\mathbb{P}}<0.01\right) & \left.=0.63 \pm 0.07 \text { (stat. })_{-0.06}^{+0.04} \text { (syst. }\right) \pm 0.03 \text { (p.d.) nb. }
\end{aligned}
$$

The last uncertainty is due to the subtraction of the proton-dissociative background.

The differential cross section as function of $x_{\mathbb{P}}$, shown in Fig. 1, demonstrates that the NLO predictions based on the various parameterisations of dPDFs are consistent with the data, supporting the validity of diffractive QCD factorisation in diffractive direct photoproduction. Differential cross sections as function of $M_{X}, p_{T}\left(D^{*}\right), \eta\left(D^{*}\right), z\left(D^{*}\right)$ and $W$ have also been calculated [2].

The ratio of the diffractive to the inclusive $\left(e p \rightarrow e D^{*} Y\right)$ photoproduction cross sections for $D^{*}$ mesons was also evaluated, as systematic uncertainties partly cancel in this ratio, which is defined as $\mathcal{R}_{\mathcal{D}}\left(D^{*}\right)=\sigma_{e p \rightarrow e D^{*} X^{\prime} p}\left(x_{\mathbb{P}}<0.035\right) / \sigma_{e p \rightarrow e D^{*} Y}$.

In the kinematic region $Q^{2}<1 \mathrm{GeV}^{2}, 130<W<300 \mathrm{GeV}(0.17<y<0.89)$, $p_{T}\left(D^{*}\right)>1.9 \mathrm{GeV}$ and $\left|\eta\left(D^{*}\right)\right|<1.6$, diffractive production for $x_{\mathbb{P}}<0.035$ contributes

$$
\left.\mathcal{R}_{\mathcal{D}}\left(D^{*}\right)=5.7 \pm 0.5 \text { (stat. }\right)_{-0.7}^{+0.4} \text { (syst.) } \pm 0.3 \text { (p.d.)\% }
$$

to the inclusive $D^{*}$ photoproduction cross section. The systematic uncertainty is dominated by the measurement of the diffractive cross section. 


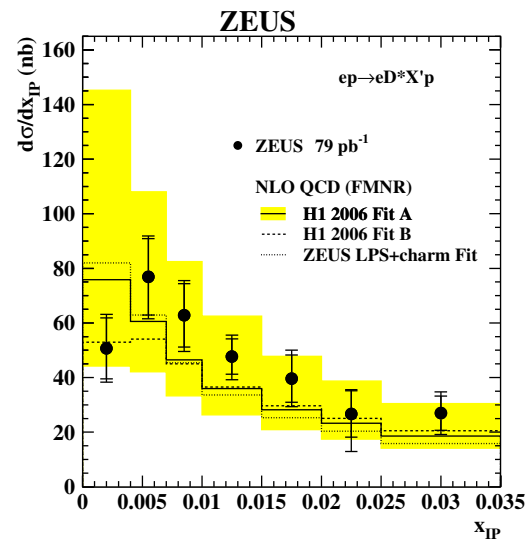

Figure 1: Differential cross section (dots) for diffractive photoproduction of $D^{*}$, measured as function of $x_{\mathbb{P}}$. The inner bars show the statistical errors; the outer bars correspond to the statistical and systematic uncertainties added in quadrature. The data are compared to the NLO QCD calculations (histograms). The shaded bands show the uncertainties coming from variations of the charm-quark mass and the factorisation and renormalisation scales.

The NLO QCD predictions for $\mathcal{R}_{\mathcal{D}}$ were obtained as the ratio of the diffractive cross section, calculated with the H1 2006 or ZEUS LPS+charm dPDFs, and the inclusive cross section, obtained with the CTEQ5M proton PDFs. The fraction $\mathcal{R}_{\mathcal{D}}$ agrees with the values measured at HERA for diffractive DIS in similar kinematic ranges $[12,3,13]$. As shown in Fig. 2, $\mathcal{R}_{\mathcal{D}}$ shows no dependence on $Q^{2}$ within the errors of the measurement. Differential distributions as function of $p_{T}\left(D^{*}\right), \eta\left(D^{*}\right), z\left(D^{*}\right)$ and $W$ have also been calculated [2].

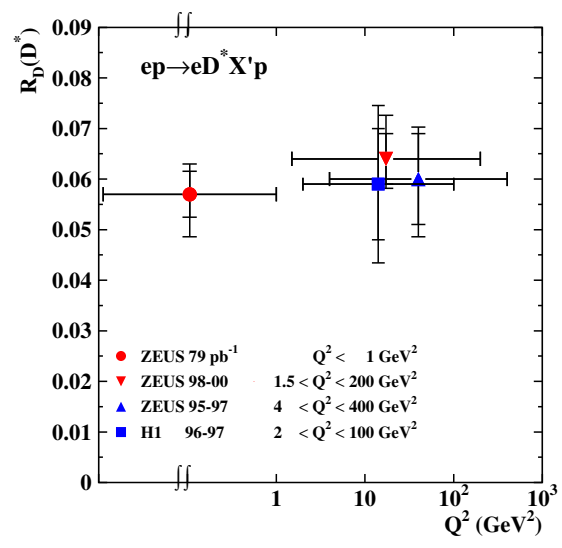

Figure 2: Fractions $\mathcal{R}_{\mathcal{D}}$ of $D^{*}$ meson diffractive production cross sections measured at HERA in DIS [3, 13, 12] and photoproduction [2]. The inner bars show the statistical errors, the outer bars correspond to the statistical and systematic uncertainties added in quadrature. 


\section{Diffractive D* in DIS}

Diffractive $D^{*}$ in DIS has been measured by ZEUS [3] in the kinematic range $1.5<Q^{2}<$ $200 \mathrm{GeV}^{2}, 0.02<y<0.7, x_{\mathbb{P}}<0.035$ and $\beta<0.8$, where $\beta$ is the fraction of the Pomeron momentum carried by the struck quark. The transverse momentum of the $D^{*}$ is restricted to $p_{T}\left(D^{*}\right)>1.5 \mathrm{GeV}$ and its pseudorapidity to $\left|\eta\left(D^{*}\right)\right|<1.5$. Using an integrated luminosity of $82 \mathrm{pb}^{-1}, 253 \pm 21 \mathrm{D}^{*}$ candidates have been found. The data have been compared to the perturbative QCD calculation program HVQDIS [14], based on a parameterisation from a gluon dominated fit to $\mathrm{H} 1$ and ZEUS inclusive diffractive DIS and ZEUS diffractive photoproduction data [15]. The data are in good agreement with the NLO predictions, confirming QCD factorisation in diffractive DIS as proven by Collins [16]. The ratio $\mathcal{R}_{\mathcal{D}}$ has been calculated as in the photoproduction analysis and is also shown in Fig 2.

\section{Conclusions}

Differential cross sections for diffractive $D^{*}$ production in photoproduction and DIS have been compared to the predictions of NLO QCD calculations. The NLO predictions based on various parameterisations of diffractive PDFs are consistent with the data. The measured fraction of $D^{* \pm}$ meson photoproduction due to diffractive exchange is consistent with the measurements of $D^{* \pm}$ meson production in diffractive DIS. Within the experimental uncertainties, this fraction shows no dependence on $Q^{2}$ and $W$.

The results demonstrate that diffractive open-charm production is well described by the dPDF parameterisations extracted from diffractive DIS data, supporting the validity of diffractive QCD factorisation in diffractive DIS and direct photoproduction. Given the large experimental and theoretical uncertainties and the small hadron-like contribution expected by the NLO calculations, no conclusion can be drawn for the resolved photoproduction.

\section{References}

[1] Slides: http://indico.cern. ch/contributionDisplay py? contribId=81\&sessionId=13\&conf Id=9499

[2] ZEUS Collaboration, S. Chekanov et al., Eur. Phys. J. C51, 301 (2007).

[3] ZEUS Collaboration, S. Chekanov et al., Nucl. Phys. B672, 3 (2003).

[4] S. Frixione et al., Nucl. Phys. B412, 225 (1994).

[5] S. Frixione et al., Nucl. Phys. B454, 3 (1995).

[6] S. Frixione et al., Phys. Lett. B319, 339 (1993).

[7] H1 Collaboration, A. Aktas et al., Eur. Phys. J. C48, 71 (2006).

[8] ZEUS Collaboration, S. Chekanov et al., Eur. Phys. J. C38, 43 (2004).

[9] W.M. Yao et al. (Particle Data Group), J. Phys. G33, 1 (2006).

[10] ZEUS Collaboration, S. Chekanov et al., Eur. Phys. J. C44, 351 (2005).

[11] C. Peterson et al., Phys. Rev. D27, 105 (1983).

[12] H1 Collaboration, C. Adloff et al., Phys. Lett. B 52, 191 (2001).

[13] ZEUS Collaboration, S. Chekanov et al., Phys. Lett. B545, 244 (2002).

[14] B.W. Harris, J. Smith, Phys. Rev. D57, 2806 (1998).

[15] L. Alvero et al, Phys. Rev. D59, 074022 (1999).

[16] J.C. Collins, Phys. Rev. D57, 3051 (1998). 